\title{
Using an aerospace monitoring for sensing "lithosphere-atmosphere-ionosphere- magnetosphere" system in order to identify and outline the potential oil and gas fields
}

\author{
Alexander Ageev ${ }^{1}$, Svetlana Bortalevich ${ }^{2}$, Evgeny Loginov ${ }^{2, *}$, Alexander Shkuta ${ }^{3}$, and \\ Dmitry Sorokin ${ }^{2}$ \\ ${ }^{1}$ RAS, Institute of Economic Strategies, 101000 Moscow, Russia \\ ${ }^{2}$ RAS, Institute of Market Problems, 117418 Moscow, Russia \\ ${ }^{3}$ Financial University under the Government of the Russian Federation, World Economics and \\ International Business Department, 125993 Moscow, Russia
}

\begin{abstract}
The article focuses on consideration of complex aerospace monitoring of aggregated territories. It is made to assess the processes took place in "lithosphere-atmosphere-ionosphere-magnetosphere" system when exploration of oil and gas fields. The research is based on the remote aerospace sounding's method. As a result of this research we have an access to the complex analysis of huge range of data. We have an opportunity to calculate a "convolution operation" in the systems of complex aerospace monitoring in order to identify the signs that can help to indicate the territory having oil and gas deposits. To sum up, the research reveals the correlation between the huge range of anomalies of the nature and the presence of oil and gas deposits in the earth on the basis of remote aerospace monitoring sounding method.
\end{abstract}

\section{Introduction}

The technical capabilities of the distant aerospace sounding of the lithosphere and atmosphere have been expanded nowadays. At the same time, new approaches to the use of collected information on the ongoing physical processes have been identified by the researchers [1].

Because of the sounding of the lithosphere-atmosphere-ionosphere-magnetosphere system we now have an opportunity to identify and outline potential oil and gas deposits.

One of the most successful methods of exploration of oil fields is the organization of the distant aerospace monitoring.

Fixing various natural anomalies allows us to allocate areas of territories with potential oil and gas deposits. We have an opportunity to calculate a "convolution operation" in the systems of complex aerospace monitoring in order to identify the signs that can help to indicate the territory having oil and gas deposits.

\footnotetext{
${ }^{*}$ Corresponding author : $\underline{\text { loginovel@mail.ru }}$
} 


\section{Problem statement}

The estimation of the geological sphere can be realized based on the analysis of various kind of data received within the framework of a permanent monitoring of the situation in the lithosphere-atmosphere-ionosphere-magnetosphere system.

For this purpose it's necessary to make tests on the prediction of electromagnetic disturbances which may took place in the lithosphere, atmosphere, ionosphere, magnetosphere and near-Earth space.

These tests results should increase the observability of the system and give the researchers an opportunity to calculate a "convolution operation" in the systems of complex aerospace monitoring in order to identify the signs that can help to indicate the territory having oil and gas deposits [2-4].

At the same time, it is necessary to reveal the correlation between the huge range of anomalies of the nature and the presence of oil and gas deposits in the earth based on remote aerospace monitoring sounding method $[5,6]$.

Various manifestations of seismic activity from individual tremors to earthquakes make the problem of predicting these very dangerous phenomena still extremely urgent.

In the last period of development of methods for remote aerospace sounding of the lithosphere and the atmosphere, the capabilities of the technical means of such sounding have expanded and, at the same time, new approaches to the use of collected information on the ongoing physical processes have emerged.

Based on the data from the sounding of the lithosphere-atmosphere-ionospheremagnetosphere system, it is possible not only to predict earthquakes, but also to identify and outline potential oil and gas deposits.

\section{Solution}

One of the ways to improve methods for predicting seismic activity and exploration of oil and gas fields is the organization of remote aerospace monitoring sounding applied to the lithosphere-atmosphere-ionosphere-magnetosphere supersystem.

According to many authoritative experts, it is the physical processes that take place not only in the lithosphere, but in the supersystem, which unites the lithosphere, atmosphere and ionosphere, form the basis for interaction of oscillatory processes, the aggregate of which programs seismic activity.

Anomalies of different physical nature, recorded in the course of sounding during complex monitoring of territories, also make it possible to identify areas of areas with potential oil and gas deposits.

It is the complex nature of monitoring that, in contrast to individual methods of oil and gas exploration, allows us for greater observability of the studied characteristics of the territory, and, consequently, to expand the range of recorded parameters of physical manifestations that indicates the presence of oil and gas.

The dynamics of monitoring the metastable states of the geo-environment and their analogues within the framework of revealing the complex structure of the factors determining seismic activity allows us to identify a set of characteristics of coherentresonant ensembles of vibrational modes as part of some transient activity of the lithosphere-atmosphere-ionosphere-magnetosphere supersystem.

Investigation of the properties of these associations (ensembles) of vibrational modes forms the possibility of predicting the exit of the geomedium beyond the limits of the quasistable states, depending on the inhomogeneity of the vibration parameters with their specific spatial configuration. 
The processes of the geomedium exit beyond the limits of the quasistable states can be modeled in the formation of self-sustaining oscillations in it with a certain spatial configuration.

Moreover, chaotic peak manifestations of seismic activity can be represented as external manifestations of complex synchronization of quasiharmonic oscillations.

It is necessary to subdivide the transitional activity of the super-system "lithosphereatmosphere-ionosphere-magnetosphere" in such a way that each of its aggregated segment from the upper mantle to the upper words of the atmosphere (taking into account the cosmic plaza) is represented as a sort of one macroobject.

This macroobject includes a number of evolving subsystems, each of which corresponds to the local state of the short-term relative equilibrium of the super-system, which can be characterized by the consolidating "convolution" of vibrational modes into ensembleforming structures.

This state of the super system is strongly or loosely coupled with the coherent-resonant ensembles of the vibrational modes and is in substantial dependence on the processes of the formation in it of self-sustaining oscillations with a certain spatial configuration.

For the forecast of seismic activity, a package of models for monitoring natural and technogenic processes is required, incl. It is necessary to estimate the state of the geomedia and the probability of its escape beyond the limits of the quasistable states.

The estimation of the probability of critical dynamics of the geo-environment can be realized on the basis of the analysis of the dynamics of functional interrelationships of various geophysical factors on the basis of a complex analysis of heterogeneous data (including temperature and humidity profiles, electron concentrations, local parameters of ionospheric plasma, infrared radiation fluxes, etc.) monitoring refinement of the dynamically changing situation in the "lithosphere-atmosphere-ionosphere-magnetosphere" system.

Here, it is necessary to operate with working parameters of complex monitoring of aggregated territories. With the corresponding processing power, the obtained information on the processes occurring in the geosphere is processed in the context of the transient activity of the lithosphere-atmosphere-ionosphere-magnetosphere supersystem.

For this purpose, it is proposed to develop a set of tests for predicting electromagnetic disturbances that can be generated both in the lithosphere and in the atmosphere, the ionosphere, the magnetosphere and near-Earth space (cosmic plasma), on the basis of retrospective analysis and the current situation.

The test results should provide an opportunity to increase the observability.

As a result, a complex solution of the problems of the structural and functional organization of the possibility of predictive operation of the bifurcation parameters of the quasistationary states of the lithosphere-atmosphere-ionosphere-magnetosphere supersystem in the realization of the interrelationships between the metastable states of the geo-environment and geophysical processes determined by anomalies of various nature (in the lithosphere, in the atmosphere, in the ionosphere, magnetosphere, etc.), within the framework of the correlation between anomalies of different physical nature and seismic activity or correlations between anomalies of different physical nature and the presence of oil and gas deposits.

At the same time, a clear understanding of the causes and identification of phases of seismic activity makes it possible to isolate the characteristics of the fixed anomalies (in the lithosphere, in the atmosphere, ionosphere, magnetosphere, etc.) that identify the presence of oil and gas deposits. 


\section{Conclusion}

As a result of this research we have an access to the complex analysis of huge range of data. There is a correlation between the geological sphere situation and the geophysical processes determined by the different natural anomalies (in the lithosphere, in the atmosphere, in the ionosphere, in the magnetosphere, etc.).

Another reviled correlation is between seismic activity, natural anomalies and the presence of oil and gas fields.

The theses were prepared with the financial support of the Russian Humanitarian Scientific Foundation (project No. 16-02-00463 a "Formation of organizational mechanisms for operating oil and gas resources on the basis of multi-agent modeling to protect Russia's economic interests from manipulating oil prices on world markets").

\section{References}

1. A. A. Makarov, T. A. Mitrova, F. V. Veselov, A. A. Galkina, V. A. Kulagin, Therm. Eng. 64(10), 703 (2017)

2. A. P. Grigorev, A. I. Soldatov, P. V. Sorokin MTT, 97 (2000)

3. D. N. Demyanovich, O. S. Vadutov, A. I. Soldatov MEACS, (2014)

4. A. S Asochakov, Y. V. Shulgina, A. I. Soldatov, E. M. Shulgin, D. N. Ogorodnikov MEACS, (2015)

5. A. S. Bugaev, E. L. Loginov, A. N. Raikov, V. N. Saraev, S\&T. Inf. Pr. 36(1), 68 (2009)

6. E. L. Loginov, A. N. Raikov, Therm. Eng. 62(4), 233 (2015) 